\title{
Die Metrik der Thaleia des Areios.
}

Nur ein einziges zusammenhängendes Stück aus der Thaleia des Areios ist der Vernichtung entgangen; aber glücklicherweise ist es ein bedeutsames, nämlich der Anfang, und ist der, der es uns mitteilt, ein Zeitgenosse des Dichters, und ist der Zweck des Zitierenden, eine Stilprobe zu geben. Die Grundlage für eine Untersuchung der Metrik ist also günstiger, als wir sie bei einem Werk erhoffen durften, dem von der Nachwelt so übel mitgespielt worden ist.

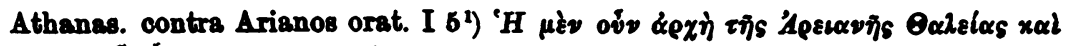
xovpohoyias hơ

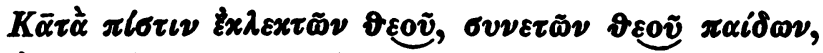

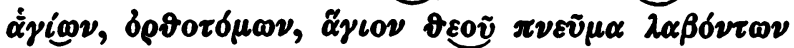

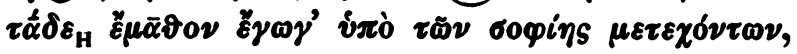

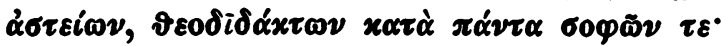

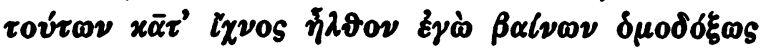

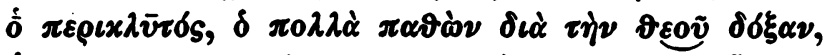

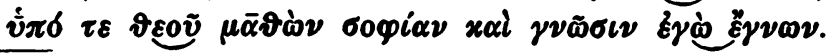

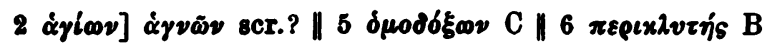

Die Zeilen hat schon Loofs ${ }^{2}$ ) abgesetzt, ohne jedoch das VersmaB zu bestimmen.

Die zweite Hälfte dieser Kola hat allemal das Metrum des Hexameterschlusses $\left(\varpi_{-} \dot{\omega_{-}}-\overline{-}_{-}-\underline{v}\right.$ ), sobald man $\vartheta \varepsilon o \tilde{v}$ einsilbig liest und in v. 7 das Augment von ž $\gamma \nu \omega \nu$ mit dem SchluBvokal von É $\gamma \dot{\omega}$ verschleift - beides Freiheiten, die wir aus der antiken Prosodie, wenn auch nicht gerade der des frühchristlichen Hexameters, belegen können. Es ergibt sich die Pflicht, die ersten Vershälften darauf anzuschauen, ob sie nicht als Hexameteranfänge lesbar sind. Ich habe die prosodischen AnstöBe, die es zu überwinden gilt, im Text bezeichnet. Neunmal muß $\breve{\iota} \breve{\iota}$ lang gemessen werden, einmal (v. 6) o; dazu kommt ein Hiatus (v. 3) und eine ungewöhnliche correptio vor muta cum liquida (v. 1). $\dot{\alpha} \gamma l @ \nu$

1) Ich habe den Coislin. 45 (C) und Burneian. 46 (B) mit dem Text der Mauriner verglichen.

2) Haucks Real-Encycl. s. v. Arianismus S. 13. 
(v. 2) kann wohl als unsicher gelten, nicht wegen der Synizese, sondern wegen des folgenden $\ddot{\gamma} \gamma\llcorner o \nu$, und vor allem, weil $\dot{\alpha} \gamma \nu \tilde{\nu} \nu$ so nahe liegt.

Was also die Prosodie dieser Zeilen von der antiken scheidet, ist im wesentlicben nur die den späteren Byzantinern ganz gelänfige Be-

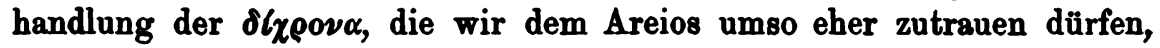
als seine vulgarisierende Tendenz fraglos ist. Ich mache noch auf die regelmäBige Paroxytonese der Versschlüsse aufmerksam.

Der Anfang der Thaleia ist also in Hexametern geschrieben. Fein sind diese Verse nicht, und besonders der erste klingt uns geradezu abscheulich. Aber Schönheit der Form rühmt kein Zeuge der Thaleia nach, und sie ist auch das letzte, was in Interesse des Areios lag. Er wollte seine Gedanken in einigermaßen gebundener Sprache unter die Masse bringen. Einen wirklich populären griechischen Vers scheint es um 320 bei den Griechen nicht gegeben zu haben: den quantitierenden Metren fügten sich die isochronen Vokale nicht, die akzentuierende Poesie war noch nicht geschaffen. So machte sich denn Areios das Versmab der Orphika and der Sibyllinen, des Parmenides und des Empedokles für seine Zwecke zurecht; und wenn das Prooimion nicht täuscht, so dankt er seinen klassischen Vorbildern noch mehr. Von seinen griechischen Zeitgenossen besitzen wir nichts Ähnliches (an die 33 prosodischen Schnitzer in dem Partheneion der Methodios mag immerhin erinnert werden); aber unter den lateinischen befindet sich einer, der den Hexameter noch freier behandelt, auch er ein Christ, vulgarisierend und dogmatisierend, Commodianus. ${ }^{1}$ )

Wir müssen natürlich auch die übrigen Fragmente der Thaleia auf ihre Metrik prüfen; ich will vorausschicken, dab dabei nicht viel herauskommt. Die ausführlichsten Zitate enthält die Sammlung der

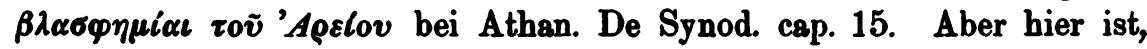
im Gegensatz zu der oben ausgeschriebenen Partie, der Zweck des Athanasios, die Dogmatik des Areios darzustellen; Zuverlässigkeit im Wortlant ist also hier nicht zu erhoffen. Die ersten 32 Zoilen sind zusammenhanglose Exzerpte, deren längstes 3 Zeilen miBt; um sie aus dem poetischen Text des Originales loszulösen, hat Athanasios zweifellos Anderungen vornehmen müssen. Mit $\Sigma v \nu \varepsilon \lambda \sigma_{\nu \tau \iota} \varepsilon i \pi \varepsilon i \nu$ (Z. 33) beginnt eine bis zum SchluB des Abschnittes verlaufende inhaltlich zusainmenhängende Partie, mit der Athanasios, wie es scheint, einen größeren Teil der Ausführungen des Areios zusammenfaBt; dies Stück darf also zunächst beiseite bleiben. Ich gebe den Text, indem ich die Zeilen gemäß den Sinnespausen absetże.

1) Jülicher (Pauly-Wissowas R. E. 8. v.) datiert ihn zwischen 250 und 350. 


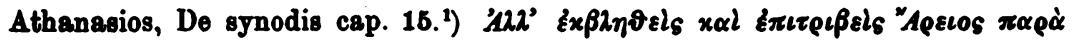

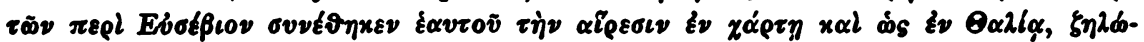

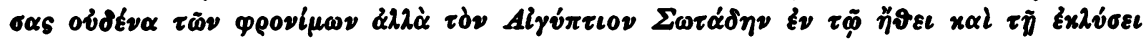

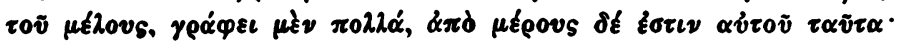

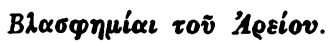

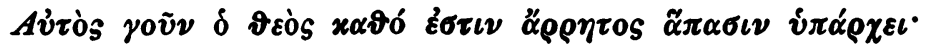

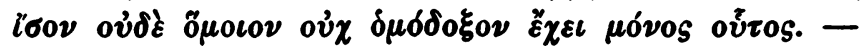

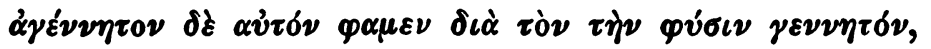

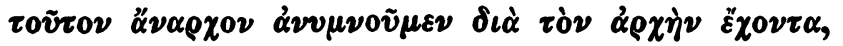

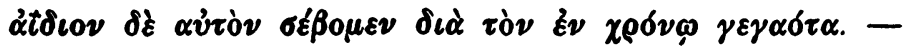

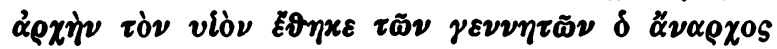

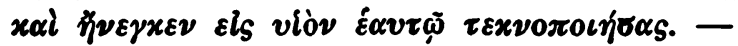

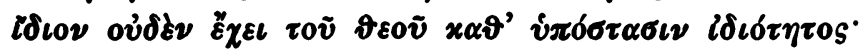

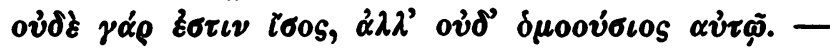

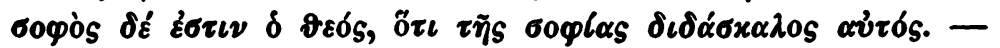

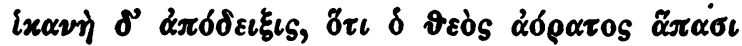

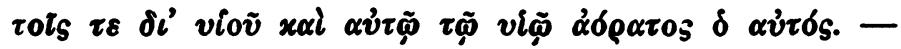


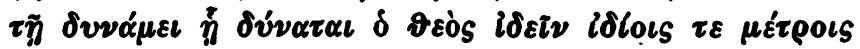

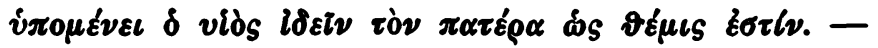

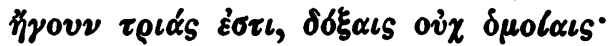

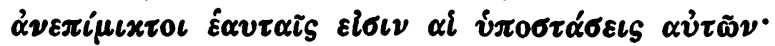

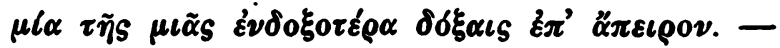

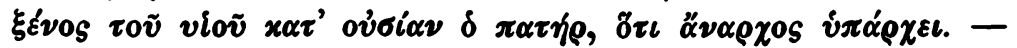

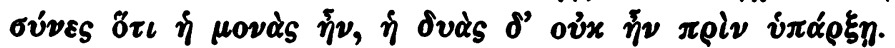

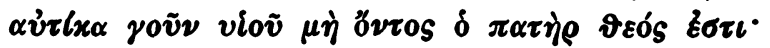

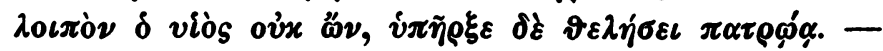

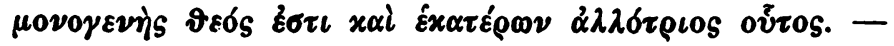

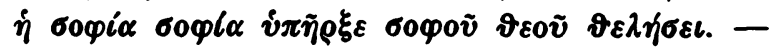

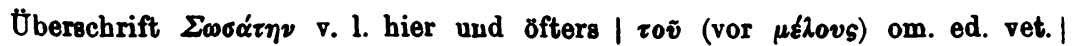

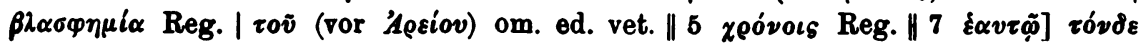
add. Reg. und ed. nov. $\| 8$ cf. Athan. contru Arian. or. 1 cap. 6 ó hójos didótelos

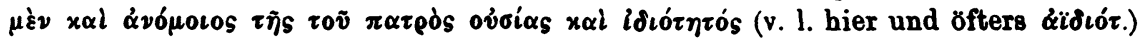

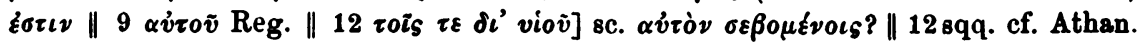

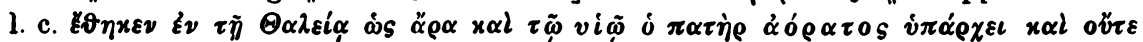

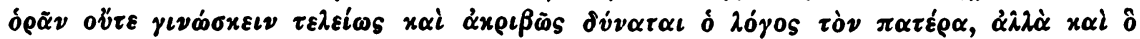

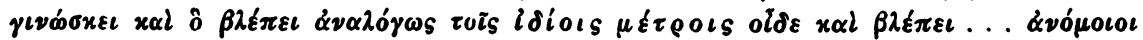

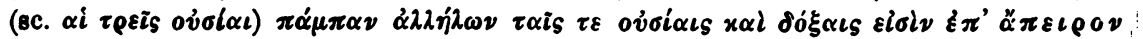

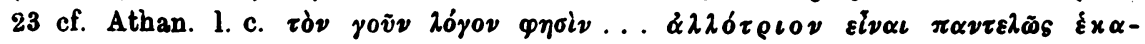

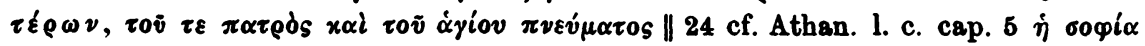

1) Von dem im Apparat aus der Ausgabe der Mauriner mitgeteilten Collationen habe ich die des Paris. gr. 474 (Reg.) selbst revidiert. Der Text ist teilweise schwer verderbt. 


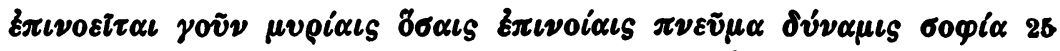

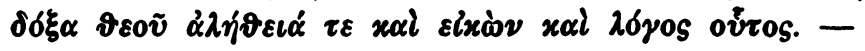

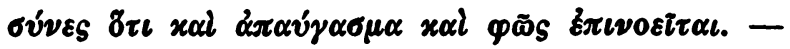

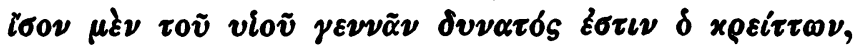

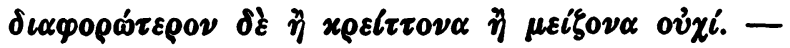

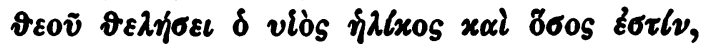

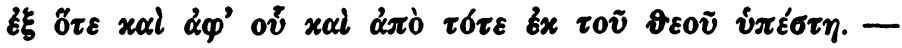

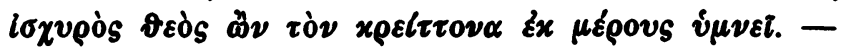

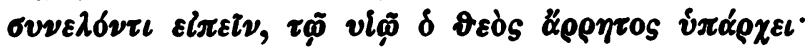

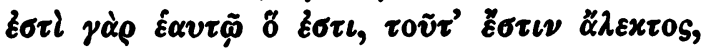

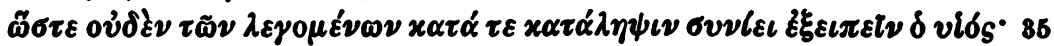

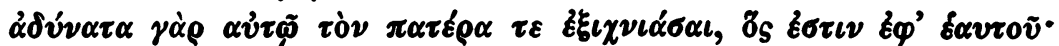

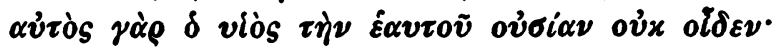

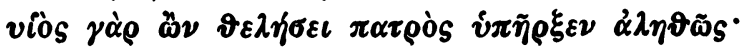

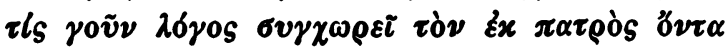

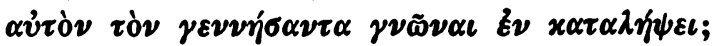

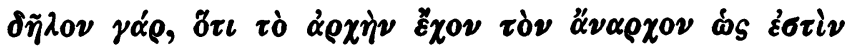

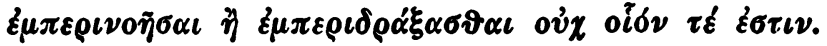

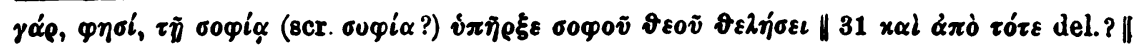

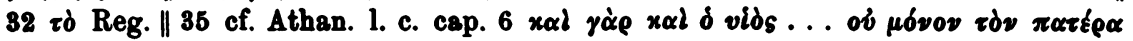

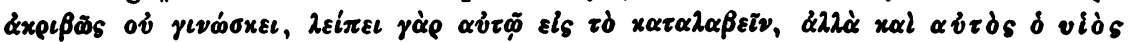

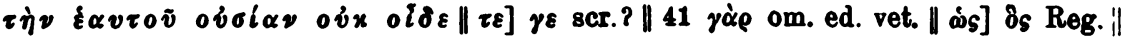
$\$ 2$ olós $\tau \varepsilon$ Reg.

In der Strecke von 1-32 sind fast alle Kola 15-20 Silben lang; nur die Sätze Z. 14-15 und 25-26 zeigen keinen klaren Einschnitt. Schon diese äuBerliche Gleichheit der Zeilen läBt auf gebundene Sprache schließen.

11 Zeilen haben den HexameterschluB: 1. 2.6 (Hiat). 7.8 (

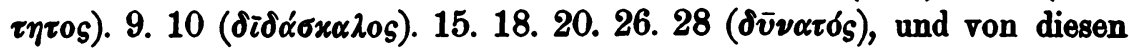

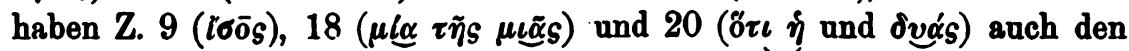
zugehörigen ersten Teil. 21 ist ein Hexameter, wenn man $\mu \eta$ ov $\nu \tau \bar{o} s$ liest. Mit leichten Streichungen - Zusätze durch Athanasios sind sehr wahrscheinlich, s. o. - kann man noch hier und da einen Vers herstellen. Aber erstens schweben solche Rekonstruktionen immer in der Luft, zweitens bedarf es keines weiteren Beweises, daB auch in den hier zitierten Teilen der Thaleia Hexameter vorhanden waren, und endlich ist es doch unmöglich, alle Exzerpte in dies VersmaB umzugieBen: Z. 2-5, durch die Parallelismen gegen den Verdacht starker Umarbeitung geschütat, und Z. 24, die ein anderes Zitat wörtlich bestätigt, sind Prosa.

Die Schlußpartie (Z. 33-42) enthält nur ganz wenig metrische 
Bestandteile und verrät nirgends disiecti membra poetae. Das Gleiche gilt für die Exzerpte bei Athanasios, Contra Arianos orat. I 2. 5. 6: hier hat wohl der Zitierende den Hauptanteil an der Diktion.

Es bleiben noch einige allgemein gehaltene Zeugnisse über die Thaleia zu besprechen. Athanasios erwähnt sie nie, ohne den Areios

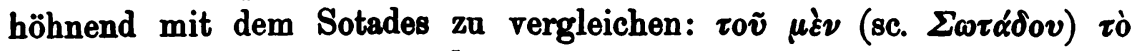

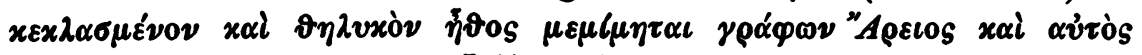

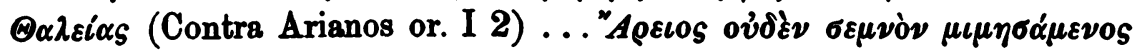

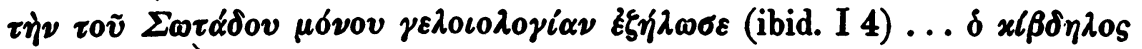

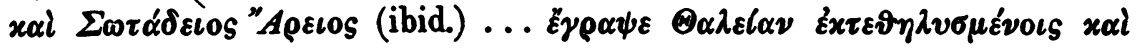

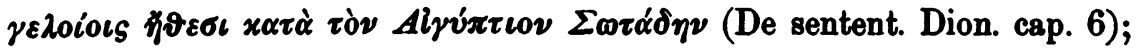
dazu die oben (S. 513) ausgeschriebene Stelle De synod. cap. 15. Von diesen Äußerungen hängt Sokrates ab, der das längst vertilgte Werk

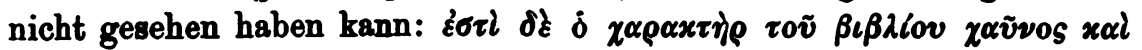

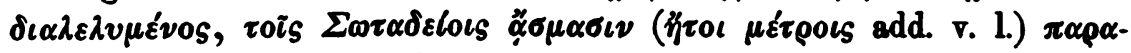
$\pi \lambda \eta \dot{\sigma c o s}$ (I 9); und den Sokrates paraphrasiert Sozomenos, mit dem

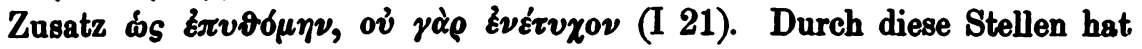
sich Loofs bewegen lassen, in den Fragmenten der Thaleia nach Sotadeen zu suchen, und Wilamowitz ${ }^{1}$ ), sie geradezu als sicher bezeugt anzusetzen. Aber Athanasios sagt das nirgends, und die Fragmente liefern keinen Sotadeus, auch nichts, was einem solchen gleichsähe. Übrigens zweifle ich, ob Areios Sotadeen dichten konnte. Von dem letzten datierbaren lebendigen Vertreter der Polymetrie, Mesomedes, ist er um zwei Jahrhunderte entfernt, von jenem Zeitpunkt, nach dem außer Hexametern, Pentametern, jambischen Trimetern und Anakreonteen kein quantitierendes $\mathrm{MaB}$ mehr auftaucht, um kaum 90 Jahre, von den ältesten quantitätslosen Gedichten, denen des Gregorios von Nazianz, nur um eine Generation. Ein so kompliziertes Metrum wie der Sotadeus fordert ein für kurze und lange Silben fühlenderes $\mathrm{Ohr}$, als das des griechisch-ägyptischen Volkes im 4. Jahrh. Was also den Areios mit dem Sotades verknüpft, muB etwas anderes gewesen sein uls das Vers-

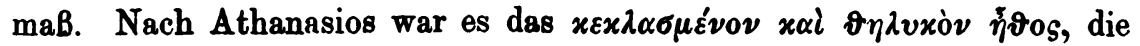

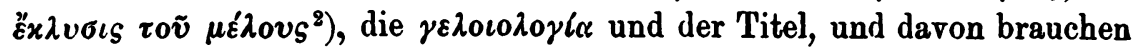
wir dem Todfeind des Areios eigentlich nur das letzte zu glauben.

München.

Paul Maas.

1) Gott. Gel. Anz. 1901, 34².

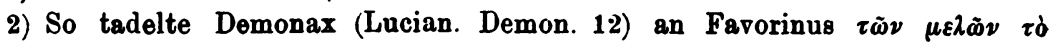

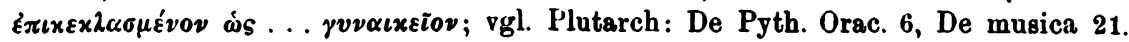
Ähnlich über das jonische Metrum, speziell den Sotadeus, Demetrios $\Pi_{\varepsilon \rho l} \varepsilon \rho \mu$. 189; Schol. in Hermog. bei Walz V 493, 6. VI 241, 25. 